\title{
Individual differences in susceptibility to visual backward masking
}

WILLIAM N. DEMBER AND ALAN NEIBERG

UNIVERSITY OF CINCINNATI
This study investigated the reliability of individual differences in susceptibility to visual backward masking. Seventeen college students were assigned "maskability" measures derived from data collected two days apart. Depending on the measures used, the rank order correlation between the two sets of measures varied from .79 to .92 , indicating highly reliable individual differences.

The perception of a visual target can be influenced by the occurrence of a subsequent visual stimulus (a "mask") under appropriate conditions. Variables that affect backward masking (the term to be used henceforth in this report) are summarized in a recent review by Raab (1963). Studies of backward masking typically have followed the psychophysical tradition of collecting large amounts of data on relatively few, and usually well-trained observers. This practice is based on the assumption that individual differences-at least among sophisticated observers-in psychophysical relations are negligible, an assumption that is difficult to test within the small-N tradition.

Scattered throughout the literature on backward masking, however, are reports of impressive individual differences (e.g., Cheatham, 1952). While such differences pose problems for data reduction, when one is seeking "the psychophysical function," they also offer possibilities for research and speculation if one is interested in individual differences as a topic of investigation.

For example, suppose observer A is highly susceptible to the masking effect, as compared with observer $B$, in the sense that $A^{\prime}$ 's recognition of a target is disrupted even when the interval between target and mask (interstimulus interval, or ISI) is long, whereas B's performance is little affected at long values of ISI. What would this difference imply about the relative efficiency of functioning of their respective visual systems? Would A be a slower processor of visual information (perhaps a less efficient reader) than $B$ ? Or, thinking of masking as an adaptive, rather than a disruptive process, would $A$, the more easily maskable observer, be more visually efficient than B? Or, if both alternatives are true; might the most efficient visual systems be possessed by those individuals who are either extremely easy or extremely hard to mask?

Questions of this sort might profitably be pursued after it is shown that there are indeed stable individual differences in susceptibility to backward masking. In the experiment reported here, such differences are clearly demonstrated. It is shown that a measure of maskability yields high test-retest reliability. That is, individual differences on this measure derived from data collected on one day a re preserved on a measure from data obtained two days later.

Method

The observer's (O's) task was to indicate which one of three letters, A, H, or $\mathbf{M}$, had been presented on a given trial. Each letter was drawn in black India ink on a white card and presented in one field of a threefield tachistoscope (Scientific Prototype, Model G). An illuminated pre-exposure field (approximately $1.8 \mathrm{ft} . \mathrm{L}$ ), with four fixation points arranged in a diamond-shaped pattern, was present prior to target exposure and reappeared at the end of each trial. A trial was initiated by $O^{\prime}$ 's depressing a button after the experimenter $(E)$ said "ready."

Target duration was set at $20 \mathrm{msec}$. throughout the experiment. At a pre-determined interval (either 5, $20,35,50,65$, or $80 \mathrm{msec}$.) after target termination, the mask was presented for a duration of $100 \mathrm{msec}$. The mask consisted of a black disc, drawn in India ink on a white card, centered on the target and just encompassing it, with a diameter of approximately $45 \mathrm{~min}$. of arc on the retina. The luminance of both the mask and target fields was approximately $10 \mathrm{ft} . \mathrm{L}$.

The termination of the mask ended a trial after which $O$ reported to $E$, guessing if necessary, what letter had been presented. The value of ISI was varied randomly from trial to trial, as was the identity of the target. A total of 45 trials, 15 per letter, was run at each of the six values of ISI on each of two days separated from each other by about $48 \mathrm{hr}$.

Data from 14 Os are presented first, six males and eight females, all students in an introductory psychology course. Data were collected from three additional Os, two males and one female, but could not be analyzed for determining reliability by the method described immediately below because they showed insufficient masking at the shortest value of ISI.

Results

The maskability measures were determined in the manner used to obtain psychophysical thresholds from data collected by the constant stimulus method. Raw frequencies of correct recognition were converted to percentages, and these were corrected for chance guessing by the formula:

$$
\mathrm{p}=\mathrm{p}_{\mathrm{o}}-\mathrm{p}_{\mathrm{c}} / 1-\mathrm{p}_{\mathrm{c}}
$$

where $p_{o}$ is obtained percent correct and $p_{c}$ is percent correct expected by chance (in this case, .33). The 
values of $\mathrm{p}$ were plotted against ISI. To these points a smooth ogival curve was fit, "by eye," and the value of ISI at which $p=.50$ was read from the curve.

Two such "threshold" measures were available for each of the $14 \mathrm{Os}$, ranging from a low of $3 \mathrm{msec}$, to a high of 42 , with a median value of 18.5 . The stability of the individual differences so measured was assessed simply by correlating the two sets of threshold values obtained from the two days' runs. The reliability, as assessed by a Spearman rank order correlation coefficient (rho) was satisfactorily high (rho=.79) and significantly different from zero $(p<.01)$.

A second method was also used for assigning a maskability measure that did not require curve-fitting and that could be applied to the data of all $17 \mathrm{Os}$. This measure was obtained simply by expressing the total number of correct recognitions at ISI values of 5 and $20 \mathrm{msec}$, as a ratio of the number at 65 and $80 \mathrm{msec}$. (where recognition was close to perfect for all Os). Again, two such ratio values were available for each $O$. These two sets of values, when correlated, yielded a rho of .86 , for the $14 \mathrm{Os}$, and a rho of .92 for all $17 \mathrm{Os}$.

As an additional point of interest a comparison was made of the "threshold" and ratio measures of maskability. Both measures were assigned to each $O$ from the combined data of both days' trials, and the two measures were correlated, yielding a rho of .96 . Clearly the two types of maskability measure are equivalent, and since the ratio measure requires fewer data points and is simpler to obtain, its use is indicated in subsequent research.

\section{Discussion}

Having established the reliability of individual differences in susceptibility to backward masking we are faced with the more interesting problem of discovering the possible correlates of these differences. It seems reasonable to expect a relation between the maskability measure and measures of "efficiency in visual information processing." Thus, maskability should set a lower bound on the rate at which elements carrying visual information can be sequentially presented to the same retinal locus. If the rate is too fast, then item $N+1$ should prevent apprehension of item $N$. In this respect, the person who is hard to mask may be the most efficient information processor. On the other hand, as suggested earlier, backward masking may have an adaptive function, in the sense of "clearing" the visual system so as to make room for new information. In this respect, ready maskability may be an asset. Thus, once we have selected what seem appropriate measures of information processing ability, we should anticipate the possibility of non-monotonic relations with the maskability measure.

\section{References}

Cheatham, P. G. Visual perceptual latency as a function of stimulus brightness and contour shape. J. exp. Psychol., 1952, 43 369-380.

Raab, D. H. Backward masking. Psychol. Bull., 1963, 60, 118-129. 